\title{
Supporting information: Nondestructive measurements of the mechanical and structural properties of nanostructured metalattices
}

Begoña Abad ${ }^{1, \dagger}$, Joshua L. Knobloch ${ }^{1}$, Travis D. Frazer ${ }^{1}$, Jorge N. Hernández-Charpak ${ }^{1}$, Hiu Y. Cheng $^{2}$, Alex J. Grede ${ }^{2}$, Noel C. Giebink ${ }^{2}$, Thomas E. Mallouk ${ }^{2}$, Pratibha Mahale ${ }^{2}$, Nabila N. Nova $^{2}$, Andrew A. Tomaschke ${ }^{3}$, Virginia L. Ferguson ${ }^{3}$, Vincent H. Crespi ${ }^{2}$, Venkatraman Gopalan $^{2}$, Henry C. Kapteyn ${ }^{1}$, John V. Badding², Margaret M. Murnane ${ }^{1}$

${ }^{1}$ Department of Physics, JILA and STROBE NSF Science \& Technology Center, University of Colorado and NIST, Boulder, CO 80309, USA

${ }^{2}$ Department of Chemistry, Biochemistry and Materials Research Institute, Pennsylvania State University, University Park, PA 16802, USA

${ }^{3}$ Mechanical Engineering, University of Colorado, Boulder, CO 80309, USA

Silicon metalattices fabrication and structural characterization Silicon was deposited at $400^{\circ} \mathrm{C}$ using ultrahigh purity silane (99.999\%) in helium as carrier gas with pressures of $20-35 \mathrm{MPa}$. The metalattices were crystallized by thermal annealing at $800^{\circ} \mathrm{C}$ in flowing argon for 30 minutes. Electron diffraction revealed them as polycrystalline. The nickel lines were fabricated by liftoff by e-beam lithography at the Nanofabrication Laboratory at Pennsylvania State University. Samples were spin coated with ZEP 520A (Zeon Chemicals) positive tone resist diluted with an equal volume of Anisole at $3000 \mathrm{rpm}$ for 45 seconds followed by a $3 \mathrm{~min} 180{ }^{\circ} \mathrm{C}$ soft bake. They were then exposed using EBPG5200 (Raith) to a target dose of $400 \mathrm{uC} / \mathrm{cm}^{2}$ with a simple proximity correction using a $1 \mathrm{nA}$ and $20 \mathrm{nA}$ beam current at $100 \mathrm{kV}$ with the lower beam current for the smallest nickel lines. Samples were developed at $-10^{\circ} \mathrm{C}$ for 3 minutes in amyl acetate, followed by 30 seconds in 2-propanol. Nickel was evaporated using an e-beam source (Lesker Lab 18) over a total area of $150 \mu \mathrm{m}$ by $150 \mu \mathrm{m}$ at $0.1 \mathrm{~nm} / \mathrm{s}$ and $3 \times 10^{-7}$ torr for a thickness of $\approx 13 \mathrm{~nm}$. The liftoff was done in PRS-3000 at $60^{\circ} \mathrm{C}$ until clear. The structural and morphological characterization of the metalattices was performed by a Zeiss Leo 1530 scanning electron microscope (SEM) with a 
$2.5 \mathrm{kV}$ accelerating voltage. We use an atomic force microscope (AFM) to study the surface morphology with a Digital Instruments MMAFM-2 Nanoscope IIIa instrument. As shown in Figure 1c of the main text, the e-beam lithographically deposited nanolines conform to the surface of the metalattice.

Experimental details on EUV nanometrology measurements and analysis. The spot size of the pump laser is $350 \mu \mathrm{m}$ to ensure uniform heating of the measured grating area. The short height of the Ni nanolines, $13 \mathrm{~nm}$, also guarantees a uniform heating through the depth of each nanoline. To extract the frequencies present in our raw traces, we use the matrix pencil method (MPM) that employs singular value decomposition to deconvolve the oscillating and decaying parts of the signal ${ }^{1}$.

Finite element analysis (FEA) to calculate speed of sound and Young's modulus of the metalattices. The theoretical value for the surface acoustic wave dispersion for the metalattice sample was computed using the commercial finite element analysis (FEA) software, COMSOL ${ }^{2}$. We implement a unit cell of the metalattice with nominal material properties for polycrystalline silicon for the infiltrated material and fused silica for the spheres. We use Bloch boundary conditions at the boundaries of the unit cell given by:

$$
\vec{u}_{\text {destination }}=\vec{u}_{\text {source }} e^{-i \vec{k} \cdot\left(\vec{r}_{\text {destination }}-\vec{r}_{\text {source }}\right)},
$$

where $\vec{u}$ is the displacement, $\vec{k}$ is the wavevector, and $\vec{r}$ is the position at the boundary. Many eigenmodes and corresponding eigenfrequencies at each $\vec{k}$ point in reciprocal space were calculated resulting in the metalattice dispersion. By fitting a line to the linear portion of the two lowest order branches of the dispersion, we extract the compressional and shear wave velocities, $V_{l}$ and $V_{s}$ respectively, along the $\Gamma \mathrm{X}$ direction of the metalattice. Solving 


$$
\left(\frac{V_{R}}{V_{s}}\right)^{6}-8\left(\frac{V_{R}}{V_{s}}\right)^{4}+8\left\{3-2\left(\frac{V_{s}}{V_{l}}\right)^{2}\right\}\left(\frac{V_{R}}{V_{s}}\right)^{2}-16\left\{1-\left(\frac{V_{s}}{V_{l}}\right)^{2}\right\}=0
$$

for $V_{R}$ results in the surface acoustic wave velocity in the absence of nanogratings on the surface ${ }^{3}$. With a sphere diameter of $30 \mathrm{~nm}$ and pore diameter of $3.5 \mathrm{~nm}$ (coming from the overlap between the spheres and resulting in a porosity of $75.55 \%$ ), the surface acoustic wave velocity is $3523 \mathrm{~m} / \mathrm{s}$. We observed no significant change to metalattice velocity between $30 \mathrm{~nm}$ and $14 \mathrm{~nm}$ diameter spheres with the same metalattice porosity.
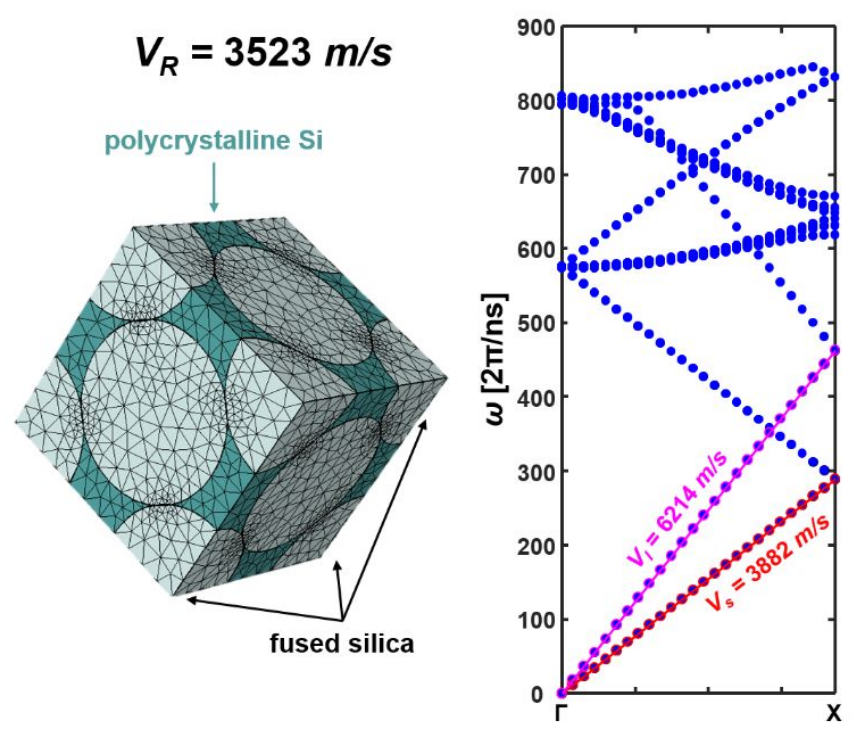

Figure S1. Metalattice structure made of polycrystalline Si infiltrated between the fused silica spheres as simulated in COMSOL (black lines show the grid of the simulation) together with the dispersion relation obtained in the $\mathrm{X}$ direction. The longitudinal (pink) and transversal (red) acoustic branches are highlighted together with their respective velocities extracted from the slope of the linear fitting.

\section{Young's modulus and Poisson ratio extraction}

From these simulations we can also calculate the Young's modulus and Poisson's ratio of the metalattice, since they are related to the transversal and longitudinal velocities calculated from the simulations as 


$$
\begin{aligned}
& V_{l}=\sqrt{(\lambda+2 \mu) / \rho} \\
& V_{s}=\sqrt{\mu / \rho}
\end{aligned}
$$

where $\rho$ is the density of the material and $\lambda$ and $\mu$ are the Lamé constants. Note that $E$ and $\sigma$ are related to $\lambda$ and $\mu$ via ${ }^{3}$ :

$$
\begin{aligned}
& \lambda=\frac{E \sigma}{(1+\sigma)(1-2 \sigma)} \\
& \mu=\frac{E}{2(1+\sigma)}
\end{aligned}
$$

\section{SAW-Likeness evaluation: experimental data fitting of Young's modulus and thickness}

Since the gratings scatter the surface acoustic wave energy into the substrate, there is no longer a solution to the wave-equation of the composite systems. Instead, we need to use SAW-likeness coefficient to identify the different modes that are propagating in our system ${ }^{4}$. This identification allows us to fit the experimentally measured frequencies to the calculated ones to find the Young's modulus from fully confined waves and thickness from waves partially travelling in the substrate.

The multilayer model includes the grating, metalattice and the silicon substrate. It assumes the metalattice as a block whose properties are used either as inputs, such as density determined from rule of mixtures, or unknown parameters that are fitted to experimental measurements.

Table S2. Metalattice properties needed for the multilayer model for SAW-Likeness evaluation.

\begin{tabular}{|c|c|}
\hline Metalattice property & How was it determined? \\
\hline Density & From rule of mixtures \\
\hline Thickness & From experiments+FEA \\
\hline Young's modulus & From experiments+FEA \\
\hline Poisson's ratio & Calculated from FEA \\
\hline
\end{tabular}




\section{Verification of filling fraction estimation}

In order to verify the estimated filling fraction of our material, we performed a series of theoretical calculations of Young's modulus for filling fractions ranging from $51 \%$ to $82 \%$ for the $30 \mathrm{~nm}$ metalattice. We then fitted the experimental frequencies for the $30 \mathrm{~nm}$ metalattice to the calculated ones from the SAW-Likeness coefficient calculation and extract the Young's modulus for each filling fraction. As we can see in Figure S2, we found that theory and experiment only agree for filling fractions in the vicinity of $76 \%$ and still lower than $80 \%$. In addition, from prior knowledge gained from SEM, the smallest filling fraction for these metalattices is $74 \%$, which corresponds to the spheres just in contact with no overlap between them. Therefore, we verify that our estimated filling fraction is correct and does not depend on the coupling between parameters. 


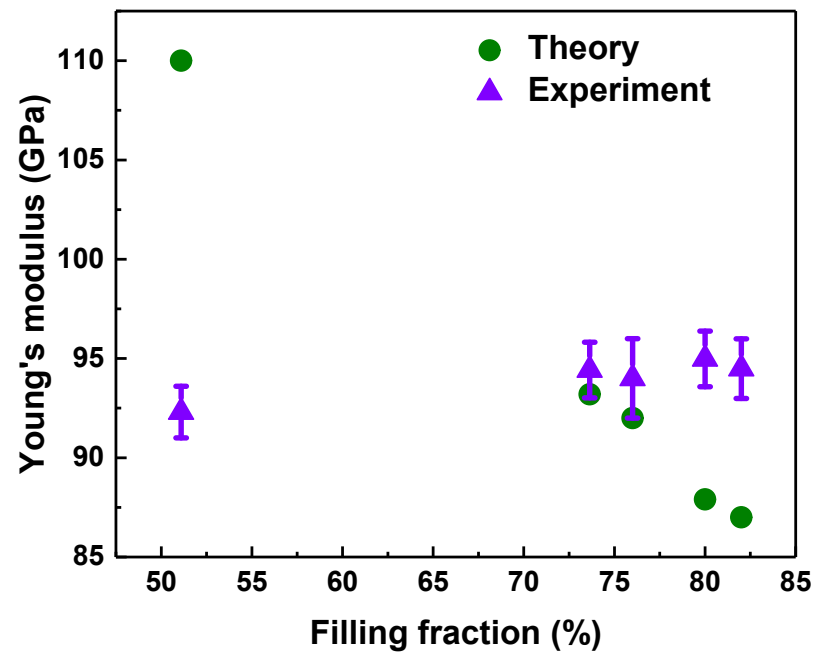

Figure S2. Young's modulus $v s$. filling fraction for both the unit cell calculation (green circles) and experiment (purple triangles). Experiments match theory for values in the vicinity of $76 \%$ which agree with estimations from fabrication and imaging characterization.

\section{Nanoindentation}

Nanoindentation tests were performed using a TI-950 Triboindenter (Bruker Nano Surfaces, Eden Prairie, MN) with a $5 \mu \mathrm{m}$ radius, conicospherical, diamond probe. Indentation arrays $(5 \times 5,5 \mu \mathrm{m}$ spacing) were set up at multiple locations on each sample. Tests were run with a trapezoidal loading scheme with a constant loading/unloading rate of $500 \mu \mathrm{N} / \mathrm{s}$, to a max load (Pmax) of 7500 $\mu \mathrm{N}$ in order to produce maximum indentation depths of approximately $10 \%$ of the film thickness. Prior to running each set of automated tests, an indent was placed into a standard fused-quartz sample to ensure that the tip was free of dirt and debris.

The load-displacement data for each test was analyzed using the OP method ${ }^{6}$ built into Bruker's Triboscan 9 software to determine a reduced modulus for each curve. Representative force-contact depth plots for each of the two materials tested can be seen in Figure S3. 


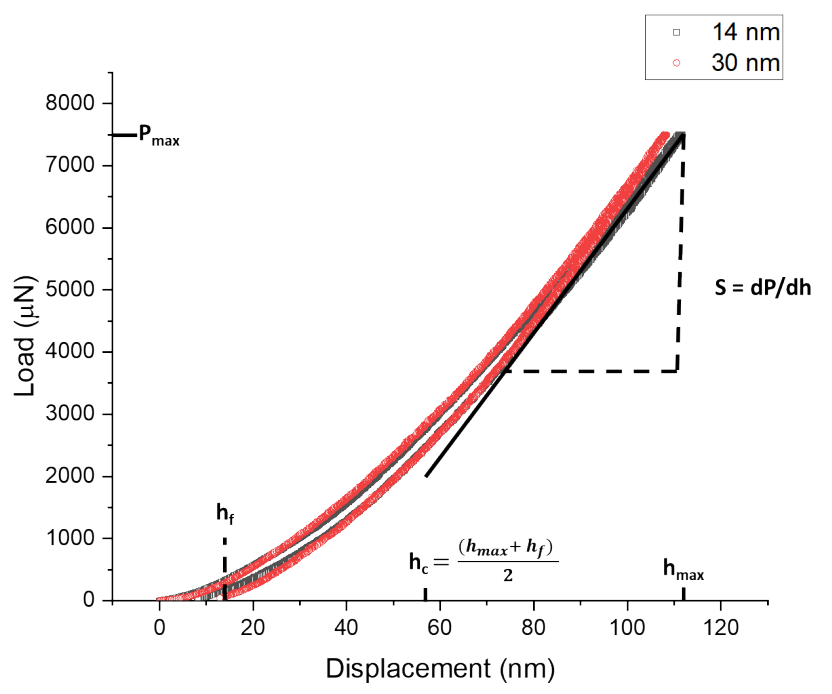

Figure S3. Representative Load vs. Displacement curves from nanoindentation of the $14 \mathrm{~nm}$ and $30 \mathrm{~nm}$ samples. The stiffness was determined from a fit of the unloading curve and used to calculate the elastic modulus according to the Oliver Pharr method.

The reduced modulus is related to the Young's modulus of the sample by:

$$
\frac{1}{E_{r}}=\frac{1-v_{i}^{2}}{E_{i}}+\frac{1-v_{s}^{3}}{E_{s}}
$$

Where $E_{r}, E_{s}$, and $E_{i}$ are the Reduced modulus, Young's modulus of the sample, and the Young's modulus of the indentation probe $\left(E_{i}=1150 \mathrm{GPa}\right.$ per Bruker Nano Surfaces $)$ and $v_{\mathrm{s}}$ and $v_{\mathrm{i}}$ are the Poisson's ratio of the sample and indentation probe $\left(v_{\mathrm{i}}=0.07\right.$ per Bruker Nano Surfaces $)$, respectively. We use the Poisson's ratio calculated from FEA simulations, 0.18. This is a good approximation since both constituent materials have similar Poisson's ratio, 0.17 and 0.22 for silica and silicon respectively. If we calculate the Young's modulus using the Poisson's ratio values of each of these values we obtain $84.99 \mathrm{GPa}$ and $83.28 \mathrm{GPa}$, respectively. These values are extremely close to the calculated for $0.18,84.68 \mathrm{GPa}$, especially considering the $10 \%$ error from the nanoindentation.

In order to ensure the analysis produced accurate data, as well as to account for any tip wear that occurred during testing, probe area function calibrations were performed at the beginning and end 
of the study. Calibrations were performed on fused-quartz with loads producing contact depths between 35 and $70 \mathrm{~nm}$, covering the range of contact depths observed during indentation of the metalattices.

Finally, in order to eliminate the influence of the substrate, a series of indentations were run with varying loads to look at the effect of the maximum indentation depth on the reduced modulus as determined by the Oliver-Pharr (OP) method. The loads ranged from $2500 \mu \mathrm{N}$ to the maximum load capacity of the transducer, $10000 \mu \mathrm{N}$, resulting in contact depths of approximately 49 to 128 $\mathrm{nm}$. The modulus as determined by the Oliver-Pharr method is plotted as a function of maximum indentation depth below and demonstrates minimal influence of the substrate (see Figure S4). Second, despite the indication that we could run indents beyond $10 \%$ of the film thickness with negligible effects, we still elected to keep our maximum depth at $10 \%$ in order to minimize substrate effects as much as possible.

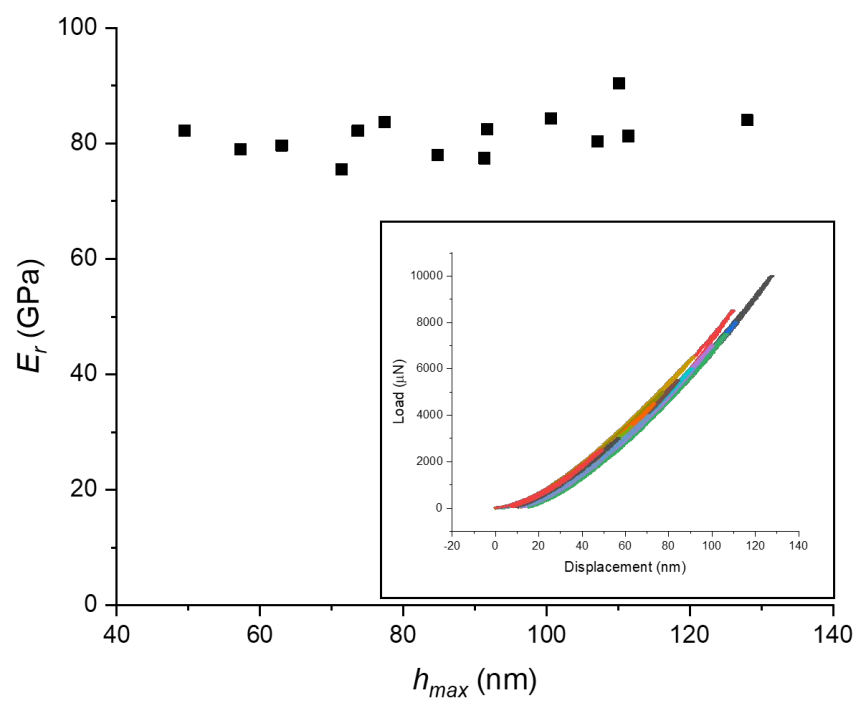

Figure S4. Plot of reduced modulus, $E_{\mathrm{r}}$, versus maximum indentation depth, $h_{\max }$, to determine the influence of substrate effects. Load was varied from $2500 \mu \mathrm{N}$ to the maximum transducer load of $10000 \mu \mathrm{N}$, producing maximum indentation depths from 49 to $128 \mathrm{~nm}$. Inset: Load versus displacement curves for the depth measurement. 
Sources of nanoindentation error: In analyzing the nanoindentation data for the metalattices, three potential sources of error were identified: surface roughness, sample mounting, and probe area function calibration.

Surface Roughness: Grau et al. ${ }^{7}$ demonstrated that hardness measurements could be collected with less than $10 \%$ standard deviation so long as the depth of the indent is 20 times the arithmetic mean surface roughness. The maximum indentation depths in this study were limited by the maximum load of the transducer and ranged from approximately 70 to $140 \mathrm{~nm}$, producing contact depths between 35 to $70 \mathrm{~nm}$, respectively. Despite the excellent surface finish of the metalattice samples, the indentation depths used would require surface roughness of $3.5 \mathrm{~nm}$ or less for the shallowest indents. In an attempt to mitigate any influence of surface roughness on the indentation measures, arrays of indents were used which allow an averaging of moduli.

Sample Mounting: To confirm that samples were robustly mounted, and to minimize error due to challenging mounting of the samples in this experiment, crystal bond was used to rigidly bond samples to a glass slide. The slide was then placed on the indenter stage. Test indents were performed in each sample to assess for inconsistencies in the load-displacement curves (e.g., relaxation during hold at max depth) that indicated a poor mounting. Data were only collected when sample mounting was confirmed to be robust and was anticipated to minimally affect indentation modulus measurements.

Probe Area Function Calibration: Calculation of modulus from depth-sensing nanoindentation relies on a well-calibrated probe area as a function of contact depth ${ }^{6}$. Here, we calibrated the probe area function using Fused Quartz (FQ), which is isotropic and which also produces indentation modulus measurements that vary by $\sim 5 \%$ (S.D.), which is lower than that of other calibration standards (e.g., single crystal aluminum S.D. $\approx 10 \%$ ). 


\section{What is the effect of Poisson's ratio on the EUV measurements?}

For both techniques, EUV nanometrology and nanoindentation, the used Poisson's ratio is assumed to be constant. Above it was clear that this was a reasonable approximation since the overall value for the Young's modulus slightly varies when using the Poisson's ratio from the constituent materials. In the case of the EUV nanometrology this is also the case. If we use these extremes for the SAW-Likeness calculations for a specific grating, we obtained results $92.39 \mathrm{GPa}$ and $94.78 \mathrm{GPa}$ for 0.17 and 0.22 , respectively. This is again close to $92.69 \mathrm{GPa}$ and within error bars.

\section{References}

(1) Hoogeboom-Pot, K. M.; Turgut, E.; Hernandez-Charpak, J. N.; Shaw, J. M.; Kapteyn, H. C.; Murnane, M. M.; Nardi, D. Nondestructive Measurement of the Evolution of LayerSpecific Mechanical Properties in Sub-10 Nm Bilayer Films. Nano Lett. 2016, 16 (8), 4773-4778.

(2) COMSOL Multiphysics® v. 5.3a. Www.Comsol.Com. COMSOL AB, Stockholm, Sweden.

(3) Auld, B. A. Acoustic Fields and Waves in Solids, Vol II; Wiley, Ed.; New York, 1973.

(4) Nardi, D.; Travagliati, M.; Siemens, M. E.; Li, Q.; Murnane, M. M.; Kapteyn, H. C.; Ferrini, G.; Parmigiani, F.; Banfi, F. Probing Thermomechanics at the Nanoscale: Impulsively Excited Pseudosurface Acoustic Waves in Hypersonic Phononic Crystals. Nano Lett. 2011, 11 (10), 4126-4133.

(5) Nardi, D.; Banfi, F.; Giannetti, C.; Revaz, B.; Ferrini, G.; Parmigiani, F. Pseudosurface Acoustic Waves in Hypersonic Surface Phononic Crystals. Phys. Rev. B - Condens. Matter Mater. Phys. 2009, 80 (10), 1-8.

(6) Oliver, W. C. Measurement of Hardness and Elastic Modulus by Instrumented Indentation: Advances in Understanding and Refinements to Methodology. J. Mater. Res. 2004, 19 (1), 3-20.

(7) Grau, P.; Ullner, C.; Behncke, H.-H. Uncertainty of Depth Sensing Hardness. Effect of Roughness. Materialprüfung 1997, 39 (9), 362-367. 
